\title{
Non-thermal radiation from interaction of compact objects with a jet in Cen A
}

\author{
Wlodek Bednarek* \\ Department of Astrophysics, University of Łódź \\ E-mail: bednarduni.lodz.pl \\ Piotr Banasiński \\ Department of Astrophysics, University of Łódź \\ E-mail: p.banasinski@uni.lodz.pl
}

\begin{abstract}
The central engines in active galaxies are immersed in huge central stellar clusters and also surrounded by spherical halos, with radii of a few tens of kpc, containing from a few hundred up to several tausend globular clusters. We investigate the acceleration of particles on the shocks formed in collisions of different compact objects at the kpc distances with jet plasma. We show that electrons can be accelerated on the shocks up to energies of the order of hundreds $\mathrm{TeV}$. They emit synchrotron radiation up to the $\mathrm{X}$-ray energies and also inverse Compton up-scatter background radiation to $\mathrm{GeV}-\mathrm{TeV}$ energies. We calculate the non-thermal radiation produced by these electrons in the jet of nearby radio galaxy Cen A.
\end{abstract}

The 34th International Cosmic Ray Conference,

30 July- 6 August, 2015

The Hague, The Netherlands

\footnotetext{
* Speaker.
} 


\section{Introduction}

The non-thermal X-ray emission from large scale jets has been discovered in two close galaxies Cen A or M 87 (Feigelson et al. 1981, Biretta et al. 1991). This X-ray emission smoothly extends up to the radio energy range supporting the idea on its common origin in the synchrotron process of $\mathrm{TeV}$ electrons (Hardcastle et al. 2001). Therefore, efficient acceleration of electrons should occur not only within the inner jet but also in the kpc scale jets. In fact, the observed X-ray knots in the intermediate scale jets seems to provide conditions for electron acceleration to $\mathrm{TeV}$ energies (e.g. Hardcastle et al. 2003). A jet, launched from the vicinity of the central supermassive black hole (SMBH), has to pass through the stellar bulge and globular cluster (GC) halo around central engine. Since the number of stars in the central stellar cluster is expected to be huge, many of them have to be immersed in the jet plasma. Shocks formed in collisions of stellar winds with the plasma in the inner jet can be also responsible for the acceleration of electrons which, comptonizing stellar radiation, might be responsible for the production of $\gamma$-ray flares (e.g. Bednarek \& Protheroe 1997, Barkov et al. 2010, Bosch-Ramon et al. 2012, Araudo et al. 2013, Wykes et al. 2014). We argue that particles, accelerated on such shocks to multi-TeV energies, produce non-thermal radiation by interacting with the magnetic field in the jet (the synchrotron radiation) and with the soft radiation from the galactic bulge at the sub-kpc distance scale and also with the Microwave Background Radiation (MBR) at the a kpc distance scale via inverse Compton process. We explore the hypothesis that relativistic electrons appear in the intermediate scale jet as a result of their acceleration on the shocks formed in collisions of many compact objects (red giants, massive stars or GCs) with the jet. More details of the model can be found in Bednarek \& Banasiński (2015).

\section{Interaction of compact objects with intermediate scale AGN jets}

Active galaxies are complicated systems surrounded at larger scale by a few important elements. The central SMBH, with the mass in the range $\sim 10^{6}-10^{10} \mathrm{M}_{\odot}$, is surrounded by the bulge, which has quasi-spherical shape, containing late type stars within the radius of the order of $\sim$ kpc. Finally, active galaxy is surrounded by a halo of GCs within the distance scale of several kpc. In the case of the nearby active radio galaxy Cen A, the massive stellar cluster has been formed around 50-100 Myrs ago as a result of a merger process with a gas reach galaxy. It has the visual dimensions of $\sim 8 \times 3 \mathrm{kpc}^{2}$ (Neff et al. 2015). The current star formation rate has been estimated on $2 \mathrm{M}_{\odot} \mathrm{yr}^{-1}$ which will result in production of $(6-12) \times 10^{7} \mathrm{M}_{\odot}$ of young stars (Wykes et al. 2014). Assuming the standard initial mass function of stars, we estimate the total number of stars with masses above $20 \mathrm{M}_{\odot}$ in the nuclear cluster in Cen $\mathrm{A}$ on $\sim 3 \times 10^{5}$. The rough estimate of the number of massive $\mathrm{O}$ and WR type stars, contained within the $1.5 \mathrm{kpc}$ from the base of the jet with the opening angle of the order of $0.1 \mathrm{rad}$ for the cluster with dimension mentioned above, is of the order of a hundred. Central regions of galaxies are also surrounded by the quasi-spherical concentrations of late type stars (galactic bulges) with typical dimensions of the order of $\sim 1 \mathrm{kpc}$ and luminosity of several percent of the total luminosity of the parent galaxy. In the case of Cen A, the number of bulge stars within the jet has been estimated on $\sim 8 \times 10^{8}$ (Wykes et al. 2014). Then the total number of red giants in Cen A jet can be as large as $\sim 10^{6}$. Also GCs are expected to enter 
the jet from time to time at the kpc distances from the base of the jet. For example, in the radio galaxy Cen A the number of GCs is estimated on 1550 (Gültekin et al. 2009). We consider active galaxy which has a jet with conical structure on the intermediate scale. The jet opening angle at a kpc distance scale is expected to be of the order of $\theta \sim 0.1 \theta_{-1} \mathrm{rad}$, see e.g. observations of the nearby active galaxy Cen A (e.g. Clarke et al. 1992, Hardcastle \& Croston 2011). We estimate the number of GCs within the jets of two well known nearby active galaxies, Cen A and M87, on $\sim 10$ and $\sim 70$ GCs for the jet opening angle equal to $0.15 \mathrm{rad}$.

The power of jets in the nearby radio galaxies are typically of the order of $\sim 10^{43} \mathrm{erg} \mathrm{s}^{-1}$ (e.g. in Cen A, Wykes et al. 2013). The ram pressure of the jet plasma can be estimated from (e.g. Bednarek \& Protheroe 1997), $P_{\mathrm{j}}=\frac{L_{\mathrm{j}}}{\pi c \theta^{2} l^{2}} \approx 1.1 \times 10^{-9} L_{43} \theta_{-1}^{-2} l_{3}^{-2}$ erg where the power of the jet is $L_{\mathrm{j}}=10^{43} L_{43} \mathrm{erg} \mathrm{s}^{-1}$, the distance from the base of the jet is $l=1 l_{3} \mathrm{kpc}$ and $c$ is the velocity of light. Compact objects, mentioned above, are also surrounded by winds which pressure can be balanced by the pressure of the jet plasma. In the case of GCs, hadronic winds from significant population of red giant stars mix efficiently with the winds from many millisecond pulsars (MSPs) within the GC. As a result, the wind emanates from the GC with the power determined by the energy output from the MSP population within the GC. We argue that particles can be accelerated at the shocks around GCs from the jet site to multi-TeV energies.

We consider collisions of stars with parameters of the red giant stars with following typical parameters, the radius $R_{\mathrm{RG}}=5 \times 10^{12} \mathrm{~cm}$, the surface temperature $T_{\mathrm{RG}}=3700 \mathrm{~K}$, the luminosity $L_{\mathrm{RG}}=10^{3} \mathrm{~L}_{\odot}$, the mass loss rate $\dot{M}_{\mathrm{w}}=10^{-7} M_{-7} \mathrm{M}_{\odot} \mathrm{yr}^{-1}$, and the wind velocity $v_{\mathrm{w}}=3 \times 10^{6} v_{3}$ $\mathrm{cm} \mathrm{s}^{-1}$. The stellar wind pressure balances the pressure of the jet plasma at the distance from the stellar surface at (Bednarek \& Protheroe 1997), $R_{\mathrm{sh}}^{\star} \approx 3.6 \times 10^{16}\left(M_{-7} v_{3}\right)^{1 / 2} \theta_{-1} l_{3} / L_{43}^{1 / 2} \mathrm{~cm}$. For small distances from the base of the jet, the pressure of the jet might not be balanced by the pressure of the wind of the red giant above its stellar surface at, $l \approx 0.15 L_{43}^{1 / 2} /\left[\theta_{-1}\left(M_{-7} v_{3}\right)^{1 / 2}\right] \mathrm{pc}$. The maximum power, that can be extracted from the jet by such a shock structure around a single star, is estimated on, $L_{\mathrm{sh}}^{\star}=L_{\mathrm{j}}\left(\frac{R_{\mathrm{sh}}^{\star}}{\theta l}\right)^{2} \approx 1.45 \times 10^{35} M_{-7} v_{3} \mathrm{erg} \mathrm{s}^{-1}$. The parameters of the massive stars are clearly much more extreme than expected for the red giants. For example, the terminal velocities of the winds of massive $\mathrm{O}$ type stars can be $v_{\mathrm{w}} \sim(1-3) \times 10^{3} \mathrm{~km} \mathrm{~s}^{-1}$ and the mass loss rates $\dot{M}_{\mathrm{W}} \sim 10^{-6} \mathrm{M}_{\odot} \mathrm{yr}^{-1}$ and of the WR type stars $\sim(1-5) \times 10^{3} \mathrm{~km} \mathrm{~s}^{-1}$ and the mass loss rates $\sim(0.8-8) \times 10^{-5} \mathrm{M}_{\odot} \mathrm{yr}^{-1}$, respectively (Lang 1992).

GCs should be also submerged in the jet. GCs are composed from old low mass stars which significant number, on average about $\sim 100$, is expected to be in the red giant phase. The mass loss rate in the range of $10^{-7} \mathrm{M}_{\odot} \mathrm{yr}^{-1}$ to $3 \times 10^{-5} \mathrm{M}_{\odot} \mathrm{yr}^{-1}$ is estimated from 100 red giants in GC. On the other hand, GCs contain many millisecond pulsars which has been accelerated to short periods due to the transfer of momentum from the stellar companions. These MSPs have typical surface magnetic fields of the order of $\sim 10^{8} \mathrm{G}$ and rotational periods of the order of $\sim 3$ $\mathrm{ms}$. These MSPs lose rotational energy in the form of the relativistic pulsar winds that should mix efficiently with slowly moving winds from the red giants (Bednarek \& Sobczak 2014). The location of the shock in the jet around the GC, $R_{\mathrm{sh}}$, can be estimated by comparing the pressure of the wind from the GC with the ram pressure of the jet plasma, $R_{\mathrm{sh}}^{\mathrm{GC}} \approx 0.93\left(L_{36} M_{-5}\right)^{1 / 4} \theta_{-1} l_{3} / L_{43}^{1 / 2}$ pc, where $L_{\mathrm{MSP}}=10^{36} L_{36} \mathrm{erg} \mathrm{s}^{-1}$ is the rotational energy loss rate of MSPs, $M_{\mathrm{GC}}=10^{-5} M_{-5} \mathrm{M}_{\odot}$ $\mathrm{yr}^{-1}$ is the mass loss rate by red giants within GC. The power which can be extracted by the shock 
from the jet plasma can be estimated on, $L_{\mathrm{sh}}^{\mathrm{GC}}=L_{\mathrm{j}}\left(\frac{R_{\mathrm{sh}}^{\mathrm{GC}}}{\theta l}\right)^{2} \approx 8.6 \times 10^{38}\left(L_{36} M_{-5}\right)^{1 / 2} \mathrm{erg} \mathrm{s}^{-1}$.

\section{Acceleration of electrons at the shock in the jet}

In order to estimate the parameters of particles which could be eventually accelerated in such scenario, we consider the physical conditions within the jet. The upper limit on the magnetic field at the base of the jet can be estimated by assuming that the Poynting flux $\left(L_{\mathrm{P}}\right)$ through the jet, is a part $(\mu)$ of the total jet power $\left(L_{\mathrm{j}}\right)$, i.e. $L_{\mathrm{P}}=\pi r_{\mathrm{in}}^{2} U_{\mathrm{B}} c \Gamma^{2}=\mu L_{\mathrm{j}}=10^{43} \mu L_{43} \mathrm{erg} \mathrm{s}^{-1}$, where the inner radius of the jet can be related to the SMBH mass $r_{\text {in }}=3 r_{\mathrm{Sch}}=10^{14} M_{8} \mathrm{~cm}$, the mass of the $\mathrm{BH}$ is $M_{\mathrm{BH}}=10^{8} M_{8} \mathrm{M}_{\odot}$, the energy density of the magnetic field is $U_{\mathrm{B}}=B^{2} / 8 \pi$, and $\Gamma$ is the Lorentz factor of the jet. The magnetic field at the base of the jet can be estimated by, $B_{\mathrm{b}} \approx 520\left(\mu L_{43}\right)^{1 / 2} /\left(\Gamma M_{8}\right) \mathrm{G}$. We assume that electrons are accelerated at the shock from the site of the jet plasma to energies limited by their synchrotron energy losses. Then, the maximum energy of electrons, limited by the synchrotron energy losses, are $E_{\mathrm{syn}} \approx 55\left(\frac{\xi}{B}\right)^{1 / 2} \approx 135 \frac{\left(\xi_{-3} \theta_{-1} l_{3} \Gamma\right)^{1 / 2}}{\left(\mu L_{43}\right)^{1 / 4}}$ $\mathrm{TeV}$, where $\xi=10^{-3} \xi_{-3}$ is the acceleration coefficient. The maximum energies of electrons can be also limited by their advection time scale along the shock. The limit on the maximum energies of electrons accelerated in the stellar shock, $E_{\mathrm{adv}}^{\star} \approx 5.6 \xi_{-3}\left(\mu M_{-7} v_{3}\right)^{1 / 2} /(\beta \Gamma) \mathrm{TeV}$, where $\beta$ is the velocity of the jet in units of the velocity of light. The power in relativistic electrons extracted from the shock, created in collision of the jet with stellar winds, is estimated on, $L_{\mathrm{e}}^{\star}=\eta N_{\star} L_{\mathrm{sh}}^{\star} \approx$ $1.45 \times 10^{40} \eta_{-1} N_{6} M_{-7} v_{3} \mathrm{erg} \mathrm{s}^{-1}$, where $\eta=0.1 \eta_{-1}$ is the energy conversion efficiency from the shock to relativistic electrons, and $N_{\star}=10^{6} N_{6}$ is the number of stars within the jet. The energy conversion efficiency, $\eta$, is usually expected to be in the range $\sim 0.1-0.2$. We constrain the maximum energies of electrons due to their advection along the shock around the GC within the jet on, $E_{\mathrm{adv}}^{\mathrm{GC}} \approx 430 \xi_{-3} \mu^{1 / 2}\left(L_{36} M_{-5}\right)^{1 / 4} /(\beta \Gamma) \mathrm{TeV}$. The total power in relativistic electrons, accelerated at the shocks around GCs, can be estimated on, $L_{\mathrm{e}}^{\mathrm{GC}}=\eta N_{\mathrm{GC}} L_{\mathrm{sh}}^{\mathrm{GC}} \approx 8.6 \times 10^{38} \eta_{-1} N_{1}\left(L_{36} M_{-5}\right)^{1 / 2} \mathrm{erg}$ $\mathrm{s}^{-1}$, where $N_{\mathrm{GC}}=10 N_{1}$ is the number of GCs within the jet.

We conclude that in fact, electrons can be accelerated to large energies at the shocks formed in collisions of GCs with jet plasma. They should produce synchrotron radiation extending up to $\mathrm{X}$-ray energy range and also multi- $\mathrm{TeV} \gamma$-rays by scattering radiation from the galactic bulge and the MBR.

\section{Non-thermal radiation from electrons}

We calculate expected synchrotron and IC spectra produced by electrons accelerated on the fronts of multiple shocks within the jet for different parameters describing compact objects and the jet content. It is assumed that electrons are accelerated with the differential power law spectra above some minimum energy $E_{\min }$. The spectral index equal to -2 is selected as expected in the shock acceleration scenario. These electron spectra have been normalized to the power transferred to electrons from the shocks in the jet. In these example calculations, we assume that the jet is semi-relativistic, with the apparent velocity equal to $\beta_{\text {app }}=0.6$ and moves at relatively large angle to the observer's line of sight estimated on $\alpha=50^{\circ}$ in the case of Cen A.

In the calculations we assume that electrons are accelerated on shocks around stars entering uniformly the jet between $10 \mathrm{pc}$ up to $1 \mathrm{kpc}$. The parameters of stars have been fixed on $\dot{M}_{\mathrm{w}}=10^{-7}$ 

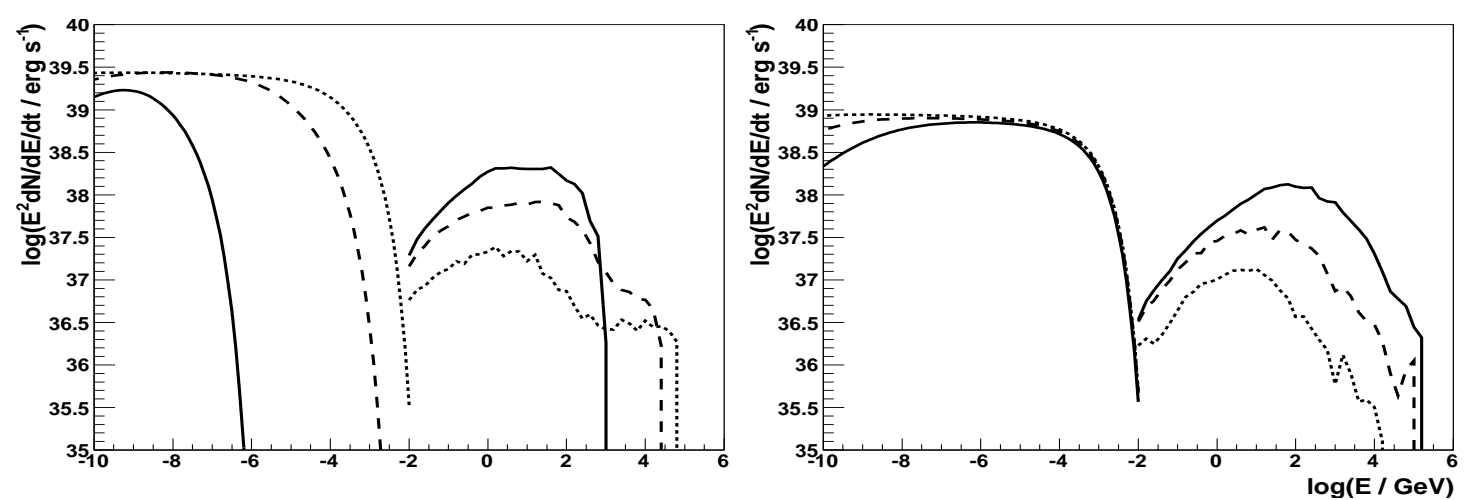

Figure 1: The synchrotron and IC spectra (SED - spectral energy distribution) produced by electrons accelerated at the multiple shocks in the jet plasma around the red giants (on the left) and massive stars (on the right), which entered the jet homogeneously on the range of distances from the base of the jet, $L_{\min }=10$ pc and $L_{\max }=1 \mathrm{kpc}$. The Poynting dominated jet, for the value of magnetization parameter equal to $\mu=1$ (dotted curves)) and the matter dominated jets described by $\mu=0.1$ (dashed), and $\mu=0.01$ (solid). The acceleration parameter of electrons is equal to $\xi=10^{-2}$. Other parameters: $L_{\mathrm{j}}=10^{43} \mathrm{erg} \mathrm{s}^{-1}, \theta=0.1 \mathrm{rad}$, $10^{6}$ red giants and 100 massive stars present in the jet, energy conversion efficiency from the jet to relativistic leptons 10\% (Bednarek \& Banasiński 2015).

$\mathrm{M}_{\odot} \mathrm{yr}^{-1}$ and $v_{\star}=30 \mathrm{~km} \mathrm{~s}^{-1}$ (for the red giant stars) and on $\dot{M}_{\mathrm{w}}=10^{-5} \mathrm{M}_{\odot} \mathrm{yr}^{-1}$ and $v_{\star}=10^{3}$ $\mathrm{km} \mathrm{s}^{-1}$ (for the massive stars). Some results of these calculations of the synchrotron and IC $\gamma-$ ray spectra are shown in Fig. 1. We investigate the spectra for the Poynting flux dominated jets (the magnetization parameter of the jet $\mu=1$ ) and for the matter dominated jets (described by $\mu=0.1$ and 0.01 ). In all considered cases, the synchrotron spectra dominate over the IC spectra. As expected, the IC $\gamma$-ray spectra are stronger for less magnetized jets. In the case of red giants, the cut-off in synchrotron spectrum is determined by the advection time scale of electrons along the shock structure.

We also calculate the synchrotron and IC spectra from collisions of GCs. We apply the parameters derived for the nearby radio galaxy Cen A: the mass of the black hole $M_{8}=0.5$, the jet power $L_{43}=1$, the jet opening angle $\theta_{-1}=1$, the jet velocity $\beta=0.5(\Gamma \approx 1.15 \sim 1)$, and the parameters of typical for GCs: the power supplied by MSPs $L_{36}=3$, the mass loss rate of red giant stars $M_{-5}=3$, and the parameters of the host galaxy bulge, the bulge stellar luminosity $L_{\text {bulge }}=10^{11} L_{\odot}$, the GC stellar luminosity $L_{\mathrm{GC}}=10^{6} L_{\odot}$, the bulge radius $R_{\text {bul }}=1 \mathrm{kpc}$. The synchrotron and IC spectra, produced by electrons accelerated at shocks formed by GCs entering the jet at the range of distances from its base between $1 \mathrm{kpc}$ and $20 \mathrm{kpc}$, are shown in Fig. 2. It is assumed that GCs enter the jet homogeneously over such range of distances and ejects electrons with the spectra and powers as described above. It is clear that in the case of the matter dominated jets the level of the $\mathrm{TeV} \gamma$-ray emission is comparable to the level of the synchrotron X-ray emission. In this case, the $\gamma$-ray spectra peak at multi-TeV energies and the synchrotron spectra extends above $\sim 10 \mathrm{keV}$. However, in the case of Poynting dominated jets, the synchrotron emission clearly dominates over the $\mathrm{TeV} \gamma$-ray emission. The $\gamma$-ray luminosity is expected to be about an order of magnitude below the $\mathrm{X}$-ray luminosity and the $\gamma$-ray spectra peak at $\mathrm{GeV}$ energies.

We apply this model for the closest radio galaxy Centaurus A (distance $3.8 \pm 0.1 \mathrm{Mpc}$ ), asso- 

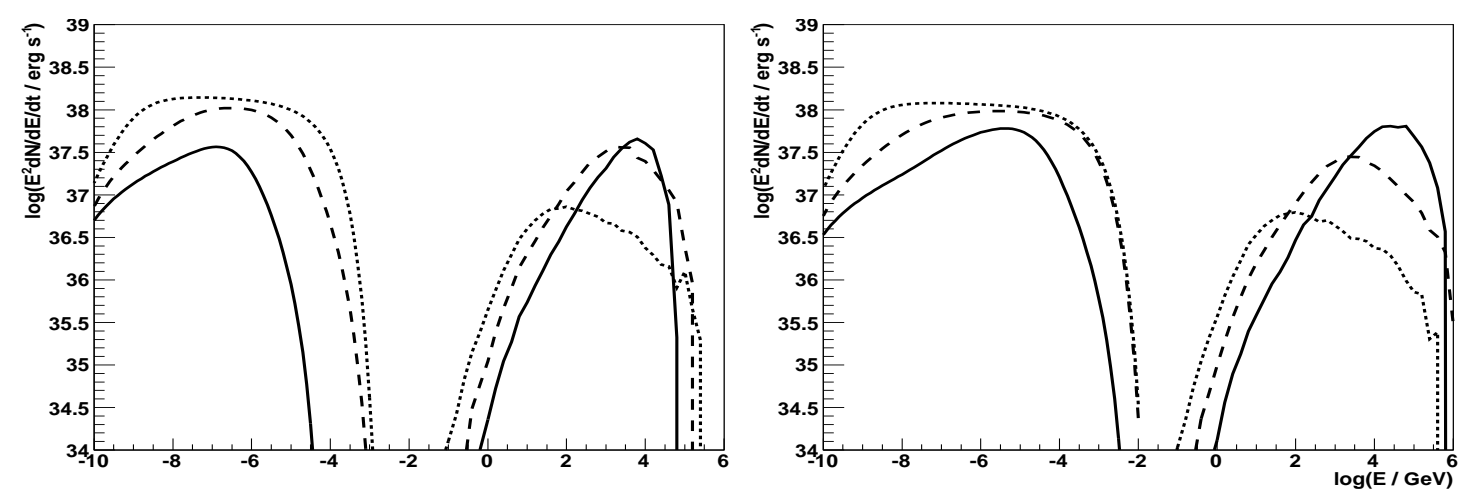

Figure 2: As in Fig. 1 but for the case of GCs entering the jet at the range of distances, $L_{\min }=1 \mathrm{kpc}$ and $L_{\max }=20 \mathrm{kpc}$. The Poynting dominated jet, for magnetization parameter equal to $\mu=1$ (dotted curves) and the matter dominated jets for $\mu=0.1$ (dashed), and $\mu=0.01$ (solid). The spectra are for the acceleration parameter of electrons equal to $\xi=10^{-3}$ (on the left) and $\xi=10^{-2}$ (on the right) (Bednarek \& Banasiński 2015).

ciated with the elliptical galaxy NGC 5128 (Harris et al. 2010). The SMBH in Cen A has the mass estimated on $M_{\mathrm{BH}}=(5.5 \pm 3.0) \times 10^{7} \mathrm{M}_{\odot}$ (Cappellari et al. 2009). The viewing angle of the jet has been estimated on $\sim 50^{\circ}$ (Tingay et al. 1998, Hardcastle et al. 2003). The power of the jet has been estimated on $P_{\mathrm{j}} \sim 10^{43} \mathrm{erg} \mathrm{s}^{-1}$ (Wykes et al. 2013). Due to its proximity, X-ray emission is clearly seen from the kpc scale jet in Cen A (Feigelson et al. 1981). At the distance between $\sim 1-3$ kpcs, the Chandra observations (0.4-2.5 keV energy range) show that the diffusive X-ray emission dominates over X-ray emission from the knots. Recently, a point like $\gamma$-ray source with the power low spectrum (spectral index $\sim 2.7$ ) extending to $\sim 2-3 \mathrm{GeV}$ has been detected by Fermi satellite from the core of Cen A (Abdo et al. 2010a). The analysis of the 4 years of the Fermi data shows that $\gamma$-ray emission extends up to $\sim 50 \mathrm{GeV}$. However, the spectrum flattens above $\sim 4 \mathrm{GeV}$ (Sahakyan et al. 2013). This flattening of the $\gamma$-ray spectrum is consistent with the detection of the TeV source towards Cen A by the HESS Collaboration (Aharonian et al. 2009). The lower energy points of these $\mathrm{TeV}$ observations $(>250 \mathrm{GeV}$ ) links nicely to the hard high energy component detected by Fermi but the spectral index of the $\mathrm{TeV}$ emission is again better described by a steep spectrum (spectral index $2.73 \pm 0.45_{\text {stat }} \pm 0.2_{\text {syst }}$, see Aharonian et al. 2009).

In order to test the hypothesis on the origin of $\gamma$-ray emission in intermediate jet, we performed calculations of the synchrotron and the IC spectra, applying the known parameters of the jet and the surrounding medium of Cen A. The results of calculations are compared with the observed high energy spectrum from Cen A (Fig. 3). Electrons are accelerated at the shocks with the energy conversion efficiency of $\eta=10 \%$. The spectra are calculated for the presence of $10^{6}$ red giants (dotted curves) and 200 massive stars (dashed curves) within the jet. For the considered parameters of the acceleration model $\left(\mu=0.01, \xi=10^{-3}\right)$, the emission from electrons accelerated on the shock around red giants is not expected to contribute significantly to the high energy spectrum observed from Cen A. On the other hand, about a hundred of massive stars in the jet can produce synchrotron emission on the level observed from the jet of Cen A. The accompanying $\gamma$-ray emission, from the componization of background radiation by these same electrons, is on the level of the $\mathrm{TeV} \gamma$-ray emission reported by HESS from Cen A. We also show the synchrotron and IC spectra produced in 


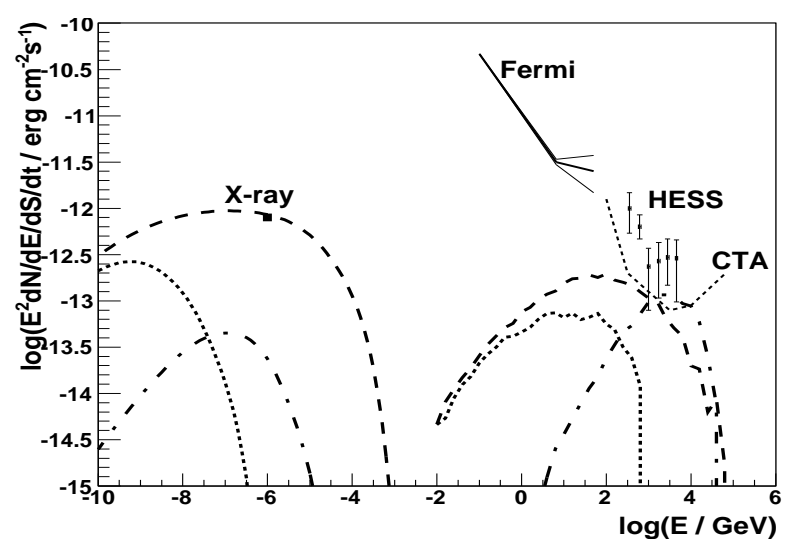

Figure 3: The comparison of the high energy observations of Cen A jet with the calculations of the synchrotron and IC spectra expected from the collisions of GCs and stars with the jet in Cen A. The X-ray emission from the kpc scale jet in Cen A from Hardcastle et al. (2006), the Fermi $\gamma$-ray spectral measurements from Sahakyan et al. (2013) and the TeV $\gamma$-ray spectrum measured by HESS from Aharonian et al. (2009). The emission expected from the interaction of $10^{6}$ red giants with the jet (dotted curves) and 200 massive stars (dashed curves) is calculated assuming that these stars enter the jet on the distance scale between $10 \mathrm{pc}$ and $1 \mathrm{kpc}$. The emission from $20 \mathrm{GCs}$ entering the jet on the distance scale between $1 \mathrm{kpc}$ and $20 \mathrm{kpc}$ (dot-dashed curves). The energy conversion efficiency equal to $10 \%$. The sensitivity of the CTA in marked by the thin dotted curve (Bednarek \& Banasiński 2015).

collisions of 20 GCs with the jet at larger distance scale (dot-dashed curves) for these same parameters of the jet. The synchrotron emission is not expected to contribute significantly to the observed $\mathrm{X}$-ray spectrum. However, the IC $\gamma$-ray emission peaks at the $\mathrm{TeV}$ energies. The cumulative $\gamma$-ray spectrum from collisions of massive stars and GCs with the jet is clearly consistent with the level of the TeV $\gamma$-ray emission observed by HESS.

\section{Conclusions}

We consider collisions of different type of objects, starting from the red giant stars from the galactic bulge, through the massive stars from the nuclear central cluster and finishing on the globular clusters in the galactic halo. Collisions produce multiple shocks in the jet on which leptons can be accelerated to $\mathrm{TeV}$ energies. These leptons are responsible for the diffusive synchrotron and inverse Compton radiation from the kpc scale jets. The high energy emission is expected to be steady during the time scales of the jet dimension (i.e. thousands of years). It is found that in general the observed level of the X-ray emission can be explained as a result of the synchrotron radiation produced by electrons accelerated on the shocks around massive stars. On the other hand, the $\mathrm{TeV} \gamma$-ray emission is expected to be produced by leptons accelerated on the shocks around massive stars (below $\sim 1 \mathrm{TeV}$ ) and by electrons accelerated on the shocks around GCs (above $\sim 1$ $\mathrm{TeV}$ ). This hypothesis could be tested in the near future with the operation of the next generation $\gamma$-ray telescopes such as CTA. CTA is expected to reach $\sim 1$ arc min resolution at $10 \mathrm{TeV}$ and the integral sensitivity of $\sim 2 \times 10^{-14} \mathrm{TeV} \mathrm{cm}^{-2} \mathrm{~s}^{-1}$ at a few TeV (Acharya et al. 2013). With such angular resolution the $\mathrm{TeV} \gamma$-ray emission from the kpc scale jet in Cen A could be clearly resolved.

This work is supported by the grant through the Polish NCN No. 2014/15/B/ST9/04043. 


\section{References}

[1] Acharya, B.S. et al. Introducing the CTA concept, APh 43 (2013) 3

[2] Aharonian, F. et al. Discovery of Very High Energy Iss-Ray Emission from Centaurus a with H.E.S.S. ApJ 695 (2009) L40

[3] Araudo, A.T. et al. Gamma rays from cloud penetration at the base of AGN jets A\&A, 522 (2010) 97

[4] Barkov, M.V. et al. Gamma-ray Flares from Red Giant/Jet Interactions in Active Galactic Nuclei, ApJ 724 (2010) 1517

[5] Bednarek, W, Banasiński, P. Non-thermal radiation from collisions of compact objects with intermediate scale jets in active galaxies ApJ in press (2015) [arXiv:1506.01181]

[6] Bednarek, W., Protheore, R.J. Gamma-rays from interactions of stars with active galactic nucleus jets, MNRAS 287 (1997) L9

[7] Bednarek, W., Sobczak, T. Misaligned TeV gamma-ray sources in the vicinity of globular clusters, MNRAS 445 (2014) 2842

[8] Biretta, J.A. et al. The radio to X-ray spectrum of the M87 jet and nucleus, AJ 101 (1991) 1632

[9] Bosch-Ramon, V., Perucho, M., Barkov, M. V. Clouds and red giants interacting with the base of AGN jets, A\&A 539 (2012) 69

[10] Clarke, D.A. et al. VLA observations of the inner lobes of Centaurus A, ApJ 395 (1992) 444

[11] Cappellari, M. et al. The mass of the black hole in Centaurus A from SINFONI AO-assisted integral-field observations of stellar kinematics, MNRAS 394 (2009) 660

[12] Feigelson, E.D. et al. The X-ray structure of Centaurus A, ApJ 251 (1981) 31

[13] Gültekin, K. et al. The M- $\sigma$ and M-L Relations in Galactic Bulges, and Determinations of Their Intrinsic Scatter, ApJ 698 (2009) 198

[14] Hardcastle, M.J. et al. Chandra observations of the X-ray jet in 3C 66B, MNRAS 326 (2001) 1499

[15] Hardcastle, M.J. et al. Radio and X-Ray Observations of the Jet in Centaurus A, ApJ 593 (2003) 169

[16] Harris, G.L.H. et al. The Distance to NGC 5128 (Centaurus A), PASA 27 (2010) 457

[17] Lang, K.R. Astrophysical Data: Planets and Stars Springer-Verlag, New York (1992)

[18] Neff, S.G., Eilek, J.A., Owen, F.N. The Complex North Transition Region of Centaurus A: A Galactic Wind, ApJ, submitted (2015) [arXiv:1502.05236]

[19] Sahakyan, N. et al. Evidence for a Second Component in the High-energy Core Emission from Centaurus A?, A\&A 770 (2013) L6

[20] Tingay, S.J. et al. The Subparsec-Scale Structure and Evolution of Centaurus A: The Nearest Active Radio Galaxy, AJ 115 (1998) 960

[21] Wykes, S. et al. Mass entrainment and turbulence-driven acceleration of ultra-high energy cosmic rays in Centaurus A, A\&A 558 (2013) A19

[22] Wykes, S. et al. Internal entrainment and the origin of jet-related broad-band emission in Centaurus A, MNRAS 447 (2015) 1001 